\title{
k-cordial Labeling of Fan and Double Fan
}

\author{
M.V. Modha ${ }^{1}$, K. K. Kanani ${ }^{2}$ \\ ${ }^{1}$ Research Scholar, School of Science, R K University, \\ Associate professor, M. D. Science College, Porbandar, Gujarat INDIA. \\ 2 Assistant Professor in Mathematics, Government Engineering College, \\ Rajkot, Gujarat INDIA.
}

\begin{abstract}
We discuss here $k$-cordial labeling of fans. We prove that fans $f_{n}$ are $k$-cordial for all $k$. We divide the proof of the result into two parts namely odd $k$ and even $k$. Moreover we prove that double fans $D f_{n}$ are $k$-cordial for all $k$ and $n=\frac{k+1}{2}$. The present authors are motivated by the research article entitled as 'A-cordial graphs' by A Hovey.
\end{abstract}

2010 Mathematics Subject Classification: 05C78

Keywords: Abelian Group; $k$-Cordial Labeling; Fan, Double fan.

\section{Introduction}

Throughout this work, by a graph we mean finite, connected, undirected, simple graph $G=(V(G), E(G))$ of order $|V(G)|$ and size $|E(G)|$.

Definition 1.1. A graph labeling is an assignment of integers to the vertices or edges or both subject to certain condition(s).

A latest survey on various graph labeling problems can be found in Gallian[2]. 
Definition 1.2. Let $\langle A, *\rangle$ be any Abelian group. A graph $G=$ $(V(G), E(G))$ is said to be $A$-cordial if there is a mapping $f: V(G) \rightarrow A$ which satisfies the following two conditions when the edge $e=u v$ is labeled as $f(u) * f(v)$

(i) $\left|v_{f}(a)-v_{f}(b)\right| \leq 1$; for all $a, b \in A$,

(ii) $\left|e_{f}(a)-e_{f}(b)\right| \leq 1$; for all $a, b \in A$.

Where

$v_{f}(a)=$ the number of vertices with label $a$;

$v_{f}(b)=$ the number of vertices with label $b$;

$e_{f}(a)=$ the number of edges with label $a$;

$e_{f}(b)=$ the number of edges with label $b$.

We note that if $A=<Z_{k},+_{k}>$, that is additive group of modulo $k$ then the labeling is known as $k$-cordial labeling.

The concept of $A$-cordial labeling was introduced by Hovey[4] and proved the following results.

- All the connected graphs are 3-cordial.

- All the trees are 3,4,5-cordial.

- Cycles are $k$-cordial for all odd $k$.

Here we consider the following definitions of standard graphs.

- The $f a n f_{n}$ is $P_{n}+K_{1}$.

- The double fan $D f_{n}$ is obtained by $P_{n}+2 K_{1}$.

\section{Main Results}

Theorem 2.1: Fans $f_{n}$ are $k$-cordial for all odd $k$.

Proof: Let $f_{n}$ be the fan and $v_{0}$ be the apex vertex. Let $n=m k+j$, where $m \geq 0$ and $1 \leq j \leq k-1$. We divide the $n$ path vertices of the fan $f_{n}$ into two blocks of $m k$ and $j$ vertices namely $v_{1}, v_{2}, . ., v_{m k}, v_{1}^{\prime}, v_{2}^{\prime}, . ., v_{j}^{\prime}$. We note that $|V(G)|=n+1$ and $|E(G)|=2 n-1$. 
Define $k$-cordial labeling $f: V(G) \rightarrow Z_{k}$ as follows.

$$
f\left(v_{0}\right)=0 ;
$$

For the first block of $m k$ vertices, if $m \geq 1$,

$$
\begin{aligned}
f\left(v_{i}\right) & =\frac{k+p_{i}}{2} ; & & i \equiv p_{i}(\bmod k), p_{i} \text { is odd }, \\
& =\frac{p_{i}}{2} ; & i & \equiv p_{i}(\bmod k), p_{i} \text { is even, } 1 \leq i \leq m k .
\end{aligned}
$$

The labeling pattern of second block of $j$ vertices, where $1 \leq j \leq k-1$ is divided into following two cases.

Case 1: $\frac{k+1}{2}$ is odd.

Sub-case I: $1 \leq j \leq \frac{k+1}{2}$.

$$
\begin{aligned}
f\left(v_{i}^{\prime}\right) & =\frac{k+i}{2} ; \quad i \text { is odd, } \\
& =\frac{k-1}{4}+\frac{i}{2} ; i \text { is even, } 1 \leq i \leq j
\end{aligned}
$$

Sub-case II: $\frac{k+3}{2} \leq j \leq k-2$.

$$
\begin{aligned}
& f\left(v_{i}^{\prime}\right)=\frac{k+i}{2} ; \quad \quad i \text { is odd, } \\
&=\frac{k-l}{4}+\frac{i}{2} ; i \text { is even, } 1 \leq i \leq j, \frac{k+l-2}{2} \leq j \leq \frac{k+l}{2}, \\
& \text { where } l=5,9, \ldots, k-4
\end{aligned}
$$

Sub-case III: $j=k-1$.

$$
\begin{aligned}
f\left(v_{i}^{\prime}\right) & =\frac{k+i}{2} ; & & i \text { is odd, } \\
& =\frac{i}{2} ; & & i \text { is even, } 1 \leq i \leq j .
\end{aligned}
$$

$\underline{\text { Case 2: }} \frac{k+1}{2}$ is even.

Sub-case I: $1 \leq j \leq \frac{k+3}{2}$.

$$
f\left(v_{i}^{\prime}\right)=\frac{k+i}{2} ; \quad i \text { is odd }
$$




$$
=\frac{k-1}{4}+\frac{i}{2} ; i \text { is even, } 1 \leq i \leq j .
$$

Sub-case II: $\frac{k+5}{2} \leq j \leq k-2$.

$$
\begin{aligned}
f\left(v_{i}^{\prime}\right) & =\frac{k+i}{2} ; \quad i \text { is odd, } \\
& =\frac{k-l}{4}+\frac{i}{2} ; \quad i \text { is even, } 1 \leq i \leq j, \frac{k+l-2}{2} \leq j \leq \frac{k+l}{2}
\end{aligned}
$$$$
\text { where } l=7,11, \ldots, k-4 \text {. }
$$

Sub-case III: $j=k-1$.

$$
\begin{aligned}
f\left(v_{i}^{\prime}\right) & =\frac{k+i}{2} ; & & i \text { is odd, } \\
& =\frac{i}{2} ; & & i \text { is even, } 1 \leq i \leq j .
\end{aligned}
$$

The labeling pattern defined above covers all possible arrangement of vertices. In each possibility the graph under consideration satisfies the vertex conditions and edge conditions for $k$-cordial labeling. Hence fans are $k$-cordial for all odd $k$.

Illustration 2.2 The fan graph $f_{23}$ and its 17-cordial labeling is shown in Figure 1.

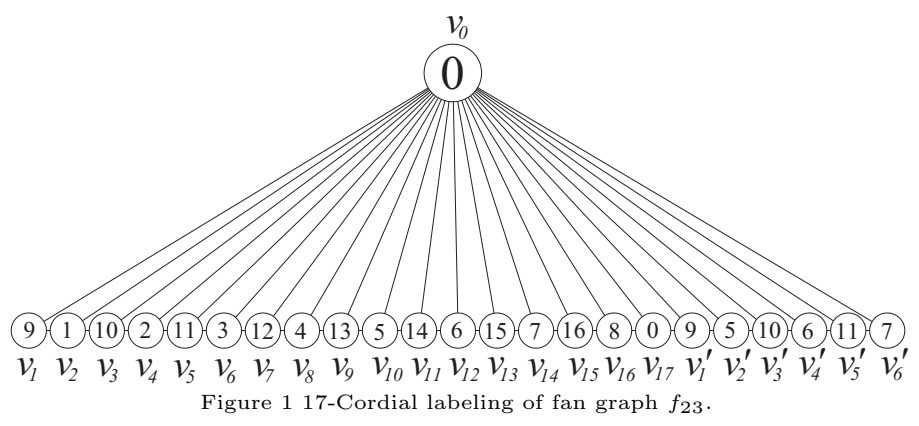

Theorem 2.3: Fans $f_{n}$ are $k$-cordial for all even $k$.

Proof: Let $f_{n}$ be the fan and $v_{0}$ be the apex vertex. Let $n=m k+j$, where $m \geq 0$ and $1 \leq j \leq k-1$. We divide the $n$ path vertices of the fan $f_{n}$ into two blocks of $m k$ and $j$ vertices namely $v_{1}, v_{2}, . ., v_{m k}, v_{1}^{\prime}, v_{2}^{\prime}, . ., v_{j}^{\prime}$. We note that $|V(G)|=n+1$ and $|E(G)|=2 n-1$. 
To define $k$-cordial labeling $f: V(G) \rightarrow Z_{k}$ we consider the following four cases.

Case 1: $m$ is odd and $\frac{k}{2}$ is odd.

$$
f\left(v_{0}\right)=0
$$

For the first block of $m k$ vertices, if $m \geq 1$,

$$
\begin{aligned}
f\left(v_{i}\right)=\frac{p_{i}+1}{2} ; \quad & i \equiv p_{i}(\bmod k), p_{i} \text { is odd, } \\
=\frac{k}{2}+\frac{p_{i}}{2} ; \quad & i \equiv p_{i}(\bmod k), p_{i} \text { is even, } \\
& (t-1) k+1 \leq i \leq t k, 1 \leq t \leq m, \text { if } t \text { is odd. } \\
f\left(v_{i}\right)=\frac{k}{2}+\frac{p_{i}+1}{2} ; & i \equiv p_{i}(\bmod k), p_{i} \text { is odd, } \\
= & \frac{p_{i}}{2} ; \quad \begin{aligned}
& \left(p_{i}(\bmod k), p_{i}\right. \text { is even, } \\
& (t-1) k+1 \leq i \leq t k, 1 \leq t \leq m, \text { if } t \text { is even. }
\end{aligned}
\end{aligned}
$$

For the second block of $j$ vertices, where $1 \leq j \leq k-1$,

Sub-case I: $1 \leq j \leq \frac{k+4}{2}$.

$$
\begin{aligned}
f\left(v_{i}^{\prime}\right) & =\frac{k}{2}+\frac{i+1}{2} ; i \text { is odd, } \\
& =\frac{k-2}{4}+\frac{i}{2} ; i \text { is even, } 1 \leq i \leq j .
\end{aligned}
$$

Sub-case II: $\frac{k+6}{2} \leq j \leq k-2$.

$$
\begin{aligned}
& f\left(v_{i}^{\prime}\right)=\frac{k}{2}+\frac{i+1}{2} ; i \text { is odd, } \\
&=\frac{k-l}{4}+\frac{i}{2} ; i \text { is even, } 1 \leq i \leq j, \frac{k+l}{2} \leq j \leq \frac{k+l+2}{2}, \\
& \text { where } l=6,10, \ldots, k-4 .
\end{aligned}
$$

Sub-case III: $j=k-1$. 


$$
\begin{array}{rlrl}
f\left(v_{i}^{\prime}\right) & =\frac{k}{2}+\frac{i+1}{2} ; & & i \text { is odd, } 1 \leq i \leq j-2, \\
& =1 ; & & i \text { is odd, } i=j . \\
& =\frac{i}{2}+1 ; & i \text { is even, } 1 \leq i \leq j .
\end{array}
$$

Case 2: $m$ is odd and $\frac{k}{2}$ is even.

$$
f\left(v_{0}\right)=0
$$

For the first block of $m k$ vertices, if $m \geq 1$,

$$
\begin{aligned}
f\left(v_{i}\right)=\frac{p_{i}+1}{2} ; \quad & i \equiv p_{i}(\bmod k), p_{i} \text { is odd, } \\
=\frac{k}{2}+\frac{p_{i}}{2} ; \quad & i \equiv p_{i}(\bmod k), p_{i} \text { is even, } \\
& (t-1) k+1 \leq i \leq t k, 1 \leq t \leq m, \text { if } t \text { is odd. } \\
f\left(v_{i}\right)=\frac{k}{2}+\frac{p_{i}+1}{2} ; i \equiv p_{i}(\bmod k), p_{i} \text { is odd, } & \\
=\frac{p_{i}}{2} ; \quad & \begin{aligned}
i & (t-1) k+1 \leq i \leq t k, 1 \leq t \leq m, \text { if } t \text { is even. }
\end{aligned}
\end{aligned}
$$

For the second block of $j$ vertices, where $1 \leq j \leq k-1$,

Sub-case I: $1 \leq j \leq \frac{k+2}{2}$.

$$
\begin{aligned}
f\left(v_{i}^{\prime}\right) & =\frac{k}{2}+\frac{i+1}{2} ; \quad i \text { is odd, } \\
& =\frac{k}{4}+\frac{i}{2} ; \quad i \text { is even, } 1 \leq i \leq j .
\end{aligned}
$$

Sub-case II: $\frac{k+4}{2} \leq j \leq k-2$.

$$
\begin{aligned}
& f\left(v_{i}^{\prime}\right)=\frac{k}{2}+\frac{i+1}{2} ; \quad i \text { is odd } \\
&=\frac{k-l}{4}+\frac{i}{2} ; \quad \quad \text { is even, } 1 \leq i \leq j, \frac{k+l}{2} \leq j \leq \frac{k+l+2}{2} \\
& \quad \text { where } l=4,8, \ldots, k-4 .
\end{aligned}
$$


Sub-case III: $j=k-1$.

$$
\begin{aligned}
f\left(v_{i}^{\prime}\right) & =\frac{k}{2}+\frac{i+1}{2} ; & & i \text { is odd, } 1 \leq i \leq j-2 \\
& =1 ; & & i \text { is odd, } i=j \\
& =\frac{i}{2}+1 ; & & i \text { is even, } 1 \leq i \leq j .
\end{aligned}
$$

Case 3: $m$ is even and $\frac{k}{2}$ is odd.

$$
f\left(v_{0}\right)=0 \text {; }
$$

For the first block of $m k$ vertices, if $m \geq 1$,

$$
\begin{aligned}
f\left(v_{i}\right)=\frac{k}{2}+\frac{p_{i}+1}{2} ; & i \equiv p_{i}(\bmod k), p_{i} \text { is odd, } \\
=\frac{p_{i}}{2} ; & i \equiv p_{i}(\bmod k), p_{i} \text { is even, } \\
& (t-1) k+1 \leq i \leq t k, 1 \leq t \leq m, \text { if } t \text { is odd } . \\
f\left(v_{i}\right)=\frac{p_{i}+1}{2} ; & i \equiv p_{i}(\bmod k), p_{i} \text { is odd, } \\
=\frac{k}{2}+\frac{p_{i}}{2} ; & \quad i \equiv p_{i}(\bmod k), p_{i} \text { is even, } \\
& (t-1) k+1 \leq i \leq t k, 1 \leq t \leq m, \text { if } t \text { is even. }
\end{aligned}
$$

For the second block of $j$ vertices, where $1 \leq j \leq k-1$,

Sub-case I: $1 \leq j \leq \frac{k+4}{2}$.

$$
\begin{aligned}
f\left(v_{i}^{\prime}\right) & =\frac{k}{2}+\frac{i+1}{2} ; i \text { is odd, } \\
& =\frac{k-2}{4}+\frac{i}{2} ; \quad i \text { is even, } 1 \leq i \leq j .
\end{aligned}
$$

Sub-case II: $\frac{k+6}{2} \leq j \leq k-2$.

$$
\begin{aligned}
f\left(v_{i}^{\prime}\right)=\frac{k}{2}+\frac{i+1}{2} ; \quad & i \text { is odd } \\
=\frac{k-l}{4}+\frac{i}{2} ; \quad & i \text { is even, } 1 \leq i \leq j, \frac{k+l}{2} \leq j \leq \frac{k+l+2}{2} \\
& \quad \text { where } l=6,10, \ldots, k-4 .
\end{aligned}
$$

Sub-case III: $j=k-1$. 


$$
\begin{aligned}
f\left(v_{i}^{\prime}\right) & =\frac{k}{2}+\frac{i+1}{2} ; & & i \text { is odd, } 1 \leq i \leq j-2, \\
& =1 ; & & i \text { is odd, } i=j \\
& =\frac{i}{2}+1 ; & & i \text { is even, } 1 \leq i \leq j
\end{aligned}
$$

Case 4: $m$ is even and $\frac{k}{2}$ is even.

$$
f\left(v_{0}\right)=0
$$

For the first block of $m k$ vertices, if $m \geq 1$,

$$
\begin{array}{rlrl}
f\left(v_{i}\right)=\frac{k}{2}+\frac{p_{i}+1}{2} ; & & i \equiv p_{i}(\bmod k), p_{i} \text { is odd, } \\
=\frac{p_{i}}{2} ; & & & i \equiv p_{i}(\bmod k), p_{i} \text { is even, } \\
& & (t-1) k+1 \leq i \leq t k, 1 \leq t \leq m, \text { if } t \text { is odd. } \\
f\left(v_{i}\right)=\frac{p_{i}+1}{2} ; & & & \\
& =\frac{k}{2}+\frac{p_{i}}{2} ; & & i \equiv p_{i}(\bmod k), p_{i} \text { is odd, } \\
& & (t-1) k+1 \leq i \leq t k, 1 \leq t \leq m, \text { if } t \text { is even. }
\end{array}
$$

For the second block of $j$ vertices, where $1 \leq j \leq k-1$,

Sub-case I: $1 \leq j \leq \frac{k+2}{2}$.

$$
\begin{aligned}
f\left(v_{i}^{\prime}\right) & =\frac{k}{2}+\frac{i+1}{2} ; \quad i \text { is odd, } \\
& =\frac{k}{4}+\frac{i}{2} ; \quad i \text { is even, } 1 \leq i \leq j .
\end{aligned}
$$

Sub-case II: $\frac{k+4}{2} \leq j \leq k-2$.

$$
\begin{aligned}
& f\left(v_{i}^{\prime}\right)=\frac{k}{2}+\frac{i+1}{2} ; \quad i \text { is odd, } \\
&=\frac{k-l}{4}+\frac{i}{2} ; \quad i \text { is even, } 1 \leq i \leq j, \frac{k+l}{2} \leq j \leq \frac{k+l+2}{2} \\
& \quad \text { where } l=4,8, \ldots, k-4
\end{aligned}
$$

Sub-case III: $j=k-1$. 


$$
\begin{aligned}
f\left(v_{i}^{\prime}\right) & =\frac{k}{2}+\frac{i+1}{2} ; & & i \text { is odd, } 1 \leq i \leq j-2, \\
& =1 ; & & i \text { is odd, } i=j . \\
& =\frac{i}{2}+1 ; & & i \text { is even, } 1 \leq i \leq j .
\end{aligned}
$$

The labeling pattern defined above covers all possible arrangement of vertices. In each possibility the graph under consideration satisfies the vertex conditions and edge conditions for $k$-cordial labeling. Hence fans are $k$-cordial for all even $k$.

Illustration 2.4 The fan graph $f_{28}$ and its 12- cordial labeling is shown in Fig 2.

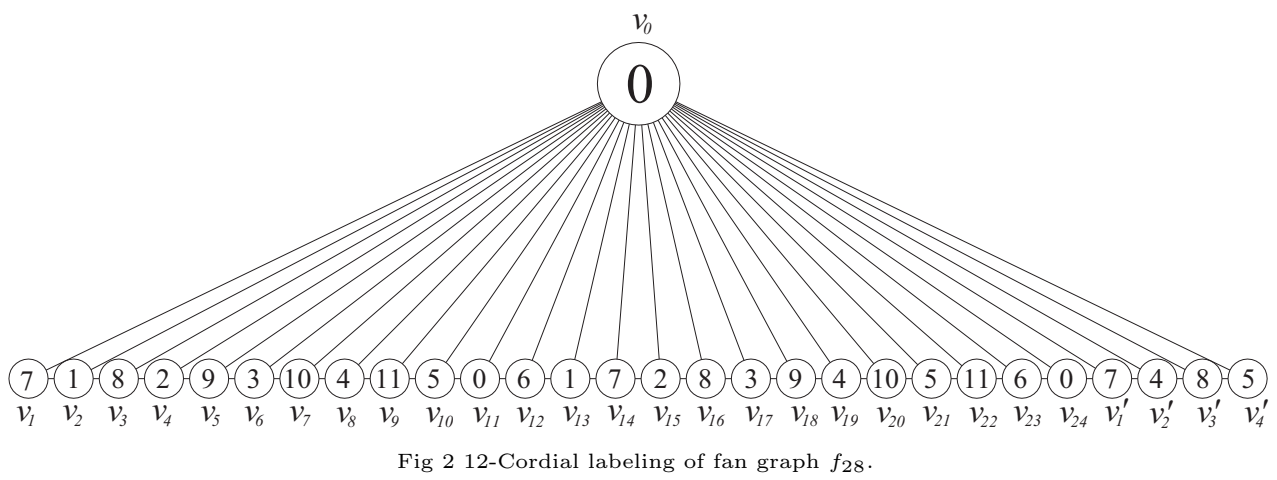

Theorem 2.5 All the double fans $D f_{n}$ are $k$-cordial for all $k$ and $n=\frac{k+1}{2}$.

Proof: Let $D f_{n}$ be the double fan. Let $v_{1}, v_{2}, \ldots, v_{n}$ be the path vertices and $v_{0}$ and $v_{0}^{\prime}$ be the two apex vertices of double fan $D f_{n}$. We note that $|V(G)|=n+2$ and $|E(G)|=3 n-1$.

To define $k$-cordial labeling $f: V(G) \rightarrow Z_{k}$ we consider the following two cases.

Case 1: $k$ is odd.

Sub-case I: $\frac{k+1}{2}$ is odd.

$$
\begin{aligned}
& f\left(v_{0}\right)=0 \\
& f\left(v_{0}^{\prime}\right)=(k-1) ; \\
& f\left(v_{i}\right)=\frac{k+i}{2} ; \quad i \text { is odd }
\end{aligned}
$$




$$
=\frac{k-1+2 i}{4} ; \quad i \text { is even, } 1 \leq i \leq n .
$$

Sub-case II: $\frac{k+1}{2}$ is even.

$$
\begin{aligned}
f\left(v_{0}\right) & =0 ; \\
f\left(v_{0}^{\prime}\right) & =(k-1) ; \quad i \text { is odd, } \\
f\left(v_{i}\right) & =\frac{k+i}{2} ; \quad \\
& =\frac{k-3+2 i}{4} ; \quad i \text { is even, } 1 \leq i \leq n .
\end{aligned}
$$

Case 2: $k$ is even.

Sub-case I: $\frac{k}{2}$ is odd.

$$
\begin{aligned}
f\left(v_{0}\right) & =0 ; \\
f\left(v_{0}^{\prime}\right) & =(k-1) ; \\
f\left(v_{i}\right) & =\frac{k-1+i}{2} ; \quad i \text { is odd } \\
& =\frac{k-2+2 i}{4} ; \quad i \text { is even, } 1 \leq i \leq n .
\end{aligned}
$$

$\underline{\text { Sub-case II: }} \frac{k}{2}$ is even.

$$
\begin{aligned}
f\left(v_{0}\right) & =0 \\
f\left(v_{0}^{\prime}\right) & =(k-1) ; \\
f\left(v_{i}\right) & =\frac{k-1+i}{2} ; \quad i \text { is odd } \\
& =\frac{k+2 i}{4}-1 ; i \text { is even, } 1 \leq i \leq n
\end{aligned}
$$

The labeling pattern defined above covers all possible arrangement of vertices. In each possibility the graph under consideration satisfies the vertex conditions and edge conditions for $k$-cordial labeling. Hence the graph of double fan admits $k$-cordial labeling.

Illustration 2.6 The double fan $D f_{22}$ and its 43-cordial labeling is shown in Fig 3. 


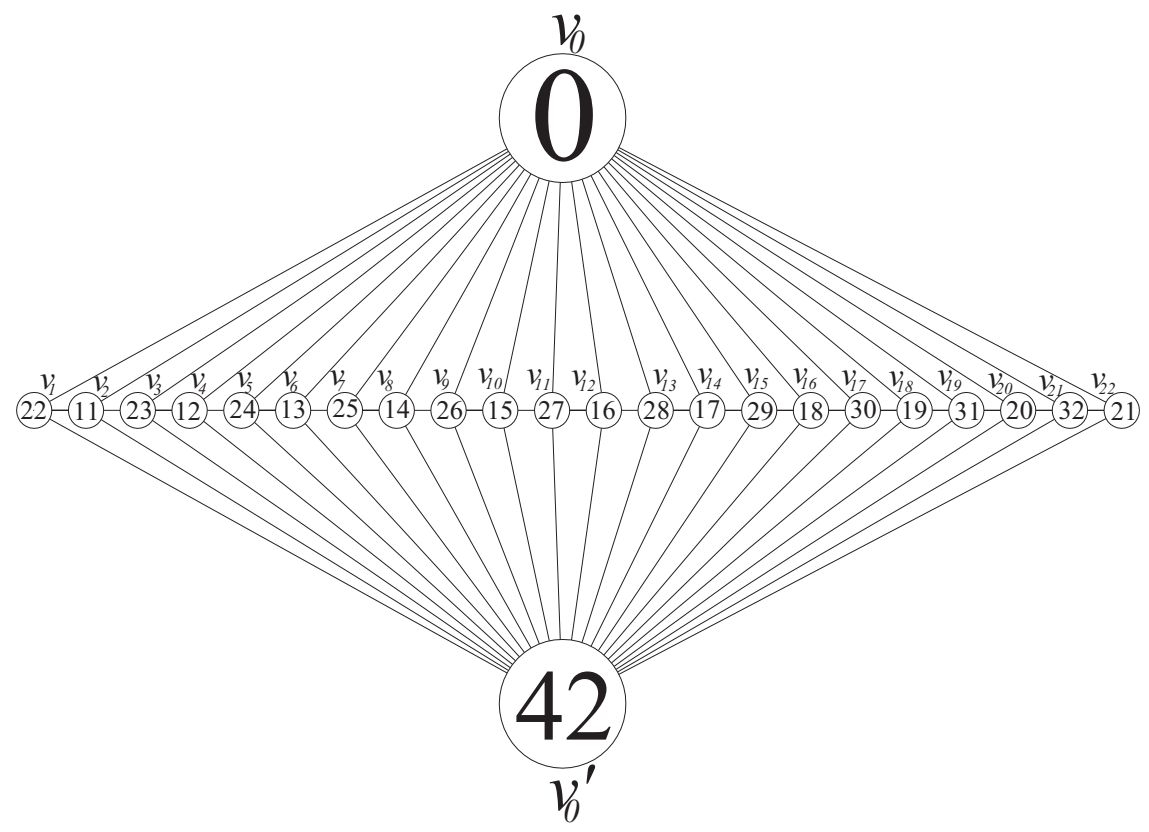

Fig 4 43-Cordial labeling of double fan $D f_{22}$.

\section{Concluding Remarks}

Here we have contributed general result for fan and a particular result for double fan to the theory of $k$-cordial labeling. To derive similar results for other graph families is an open problem.

\section{References}

[1] L. W. Beineke and S. M. Hegde, Strongly multiplicative graphs, Discuss. Math. Graph Theory,Vol 21,63-75 (2001).

[2] J A Gallian, A dynamic survey of graph labeling, The Electronics Journal of Combinatorics, 16(2014) $\sharp D S 6$.

[3] J Gross and J Yellen, Handbook of graph theory, CRC Press, (2004).

[4] M. Hovey, A-cordial graphs, Discrete Math.,Vol 93,183-194 (1991).

[5] M. Z. Youssef, On k-cordial labeling, Australas. J. Combin.,Vol 43,31-37 (2009).

[6] R. Tao, On k-cordiality of cycles, crowns and wheels, Systems Sci. Math. Sci., 11 (1998) 227-229. 